\title{
Optical Piezoelectric Transducer For Nano-Ultrasonics
}

\author{
Kung-Hsuan Lin, Gia-Wei Chern, Cheng-Ta Yu, Tzu-Ming Liu, Chang-Chi Pan, Guan-Ting Chen, \\ Jen-Inn Chyi, Senior Member, IEEE, Sheng-Wen Huang, Pai-Chi Li, Senior Member, IEEE, \\ and Chi-Kuang Sun, Senior Member, IEEE
}

\begin{abstract}
Piezoelectric semiconductor strained layers can be treated as piezoelectric transducers to generate nanometer-wavelength and THz-frequency acoustic waves. The mechanism of nano-acoustic wave (NAW) generation in strained piezoelectric layers, induced by femtosecond optical pulses, can be modeled by a macroscopic elastic continuum theory. The optical absorption change of the strained layers modulated by NAW through quantum-confined Franz-Keldysh (QCFK) effects allows optical detection of the propagating NAW. Based on these piezoelectric-based optical principles, we have designed an optical piezoelectric transducer (OPT) to generate NAW. The optically generated NAW is then applied to one-dimensional (1-D) ultrasonic scan for thickness measurement, which is the first step toward multidimensional nano-ultrasonic imaging. By launching a NAW pulse and resolving the returned acoustic echo signal with femtosecond optical pulses, the thickness of the studied layer can be measured with $<1 \mathrm{~nm}$ resolution. This nano-structured OPT technique will provide the key toward the realization of nano-ultrasonics, which is analogous to the typical ultrasonic techniques but in a nanometer scale.
\end{abstract}

\section{INTRODUCTION}

$\mathrm{M}$ EGAHERTZ to gigahertz ultrasonic technologies and their related applications have been well developed. The typical method to generate and detect acoustic waves is to apply and monitor the electric bias changes of electrodes on a piezoelectric material. However, the limitations of current methods to adopt higher acoustic frequency have to do with the difficulty in shortening the interval of electrodes and the limited bandwidth of the electronic system.

Grahn et al. [1] and Maris [2] proposed to use femtosecond laser light to generate picosecond sound pulses

Manuscript received March 31, 2004; accepted December 20, 2004. This work is sponsored by the National Science Council of Taiwan R.O.C. under Grant No. 92-2120-M-002-004, NSC-92-2112-M-002044, and NSC-92-2215-E-008-020.

K.-H. Lin, C.-T. Yu, and T.-M. Liu are with the Graduate Institute of Electro-Optical Engineering, National Taiwan University, Taipei 10617, Taiwan.

G.-W. Chern is with the Department of Physics and Astronomy, Johns Hopkins University, Baltimore, MD 21218.

G.-T. Chen, C.-C. Pan, and C.-I. Chyi are with the Department of Electrical Engineering, National Central University, Chung-Li 32045, Taiwan.

S.-W. Huang and P.-C. Li are with the Department of Electrical Engineering, National Taiwan University, Taipei 10617, Taiwan.

C.-K. Sun is with the Department of Electrical Engineering and Graduate Institute of Electro-Optical Engineering, National Taiwan University, Taipei 10617, Taiwan (e-mail: sun@cc.ee.ntu.edu.tw). for acoustic characterizations in materials, and this established technology is now called picosecond ultrasonics. By focusing a femtosecond laser light onto the surface of a material with a thin absorption layer, the energy of the photons initially will be taken up by electrons and then transferred to lattice heat, causing the layer to expand [3]. As a result, a sound wave, as short as several picoseconds and mostly a broad-band single pulse, launches into the material. When the generated acoustic pulse travels through the sample and its echo returns to the surface, it can be detected by another short optical pulse directed at the surface. Although the pulsewidth of the acoustic wave is on the scale of picoseconds, this technology still is unable to manipulate the phase of the initiated acoustic wave.

However, terahertz coherent longitudinal-acoustic (LA) phonon oscillation generated in piezoelectric semiconductor multiple strained-layer structure (such as multiple quantum wells) was recently experimentally demonstrated [4], [5], and a microscopic theory was presented later [6], [7]. Because the induced LA phonons have a high degree of coherence in time, its generation and propagation can be modeled by a macroscopic continuum elastic theory [8], [9]. This semiclassical viewpoint of the coherent LA phonon oscillation as an acoustic wave is valid in the coherent regime in which thermal and quantum fluctuations can be neglected. Because the wavelength of the acoustic wave is determined by the period of strained layers, it easily can be much less than $10 \mathrm{~nm}$. Because the acoustic wave is primarily induced through the piezoelectric effect [6]-[9], this multiple strained-layer structure (SLS) can be treated as an optical piezoelectric transducer (OPT) to generate nano-acoustic waves (NAW). Compared with a typical electrical transducer, the main difference is that the variation of the electric field in the nano-SLS OPT is induced by femtosecond laser pulses instead of electric pulses.

In this paper, we shall discuss the operation principles of the nano-structured OPT conceptually and experimentally from the viewpoint of acoustic engineering. Through a thickness measurement, we will demonstrate that the OPT with nano-structures is an ideal wave source to realize the field of nano-ultrasonics, which is analogous to the typical ultrasonics but in a nanometer scale. In Section II, we shall describe the studied sample structure. The principles of optical generation and optical detection of NAW will be presented in Sections III and IV, respectively. In Section V, we will discuss the principle of OPT design. 


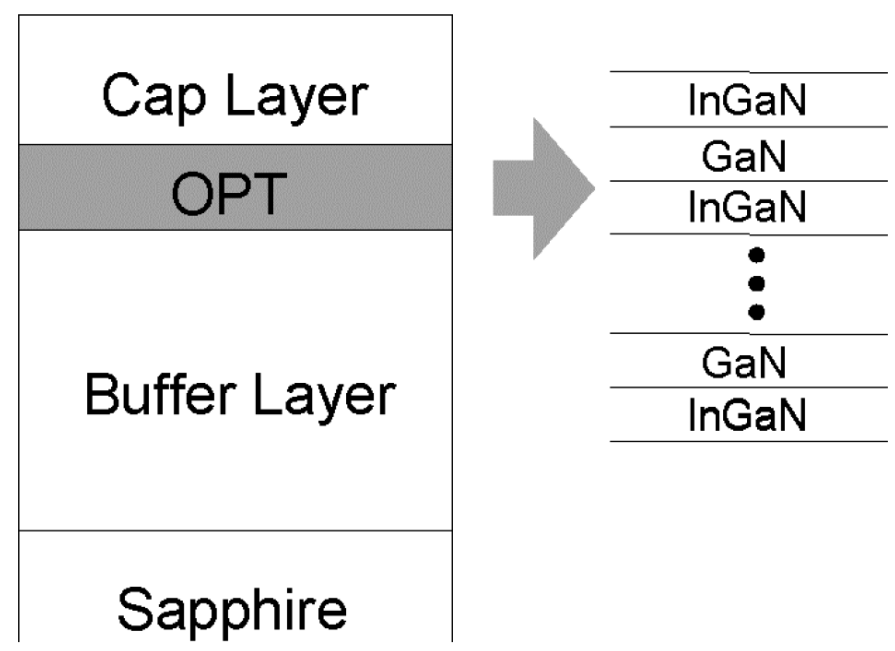

Fig. 1. Diagram of the sample structure. The periodic InGaN strained-layer region is treated as an optical piezoelectric transducer. The cap layer is the studied propagating medium.

The characterization of the OPT will be presented in Section VI. Then we shall demonstrate the transient waveform synthesis in Section VII. In Section VIII, we will demonstrate the one-dimensional (1-D) nano-ultrasonic scan for thickness measurement by using the optically generated NAW. We will discuss the advantages of this OPT technology and explore the feasibility to apply this novel acoustic device to nano-ultrasonic imaging in Section IX. Conclusions will be presented in Section $\mathrm{X}$.

\section{Preparation of SAmples}

GaN-related compounds are popular materials for ultraviolet-blue-green lasers and light emitting diodes [10]. The bandgap of GaN is $\sim 3.4 \mathrm{eV}$ corresponding to a near ultraviolet photon energy. By replacing part of gallium atoms with indium atoms, the bandgap of InGaN shifts toward longer optical wavelength (blue in our case). Besides their important roles in electronic and optoelectronic devices, GaN-related compounds are piezoelectric materials and have been applied to electric piezoelectric transducers to generate acoustic waves with a gigahertz frequency [11]. To obtain higher acoustic frequency, the key point is to achieve temporally faster and spatially narrower electric field modulations. Therefore, femtosecond laser pulses and nano-structured GaN are required.

We used piezoelectric InGaN/GaN multiple strained layers as the nano-structured OPT for the generation of NAW. Fig. 1 shows a basic structure of the samples to be discussed in this paper. The InGaN/GaN samples were grown by metal organic chemical vapor deposition (MOCVD) on top of a GaN buffer layer with a thickness of more than $2 \mu \mathrm{m}$ on c-plane sapphire substrates [12], [13]. Then a cap layer is grown on top of the OPT. The cap layer is the studied propagating layer because it is much thinner than the GaN buffer layer. The nitride layers all are composed of a wurtzite structure.
In this paper, the SLS in sample $\mathrm{A}$ is a 14period $22 \AA / 70 \AA \operatorname{In}_{0.1} \mathrm{Ga}_{0.9} \mathrm{~N} / \mathrm{GaN}$ multiple quantum well (MQW). It is capped with an $\sim 100 \mathrm{~nm}$-thick $\mathrm{Al}_{0.06} \mathrm{GaN}$ cap layer. The SLS in sample B is a 3 -period $29 \AA / 130 \AA$ $\mathrm{In}_{0.23} \mathrm{Ga}_{0.77} \mathrm{~N} / \mathrm{GaN}$ MQW. It is capped with an $\sim 80-\mathrm{nm}$ thick GaN cap layer. Detailed growth conditions have been reported elsewhere [12], [13].

\section{Generation of NAnO-Acoustic Waves}

To initiate NAW, we have used the optically excited carriers to screen the existing electric field distribution in the SLS OPT. We shall first discuss the equilibrium strain-induced electric field distribution in the SLS OPT. The epitaxial technology nowadays allows us to grow one material on another up to an atomic accuracy. We call the semiconductor with wider bandgap barrier and the other quantum well (or well). Due to the lattice mismatch between the barrier and the well, the lattices of one or both materials distort near the interface (or within the relaxation thickness) to result in a strained layer. When a strain distribution takes place in a piezoelectric material, it causes a piezoelectric field through the piezoelectric effect. And the magnitude of this background electric field distribution determines the maximum strength of the NAW because we have used the screening effect to modulate the background piezoelectric field. For the case of InGaN/GaN MQW, the equilibrium piezoelectric field can be greater than $1 \mathrm{MV} / \mathrm{cm}$ [14], and thus large amplitude acoustic waves can be generated if the background piezoelectric field is completely screened.

To generate acoustic waves with a several nanometer wavelength, the thickness of the acoustic layer with a spatially modulated electric field distribution also should be on the order of several nanometers. It can be achieved easily with the matured epitaxial technology. However, for generating acoustic waves with a frequency in subterahertz to terahertz regime, the time to change the electric field should be less than 1 ps. To achieve this requirement, a femtosecond (fs) optical pulse will provide the ultrahigh frequency initiating source.

When the photon energy of the fs optical pulses is between the bandgap of the well and the barrier, carriers (such as electrons and holes) can be excited by the fs optical pulses only in the wells within the time scale of the optical pulsewidth. The photoexcited electrons and holes are immediately separated in the wells due to the builtin piezoelectric field, and they serve as the driving force to initiate strain waves in the OPT through space-charge screening. It is of note that the excited carriers all will stay in the wells due to the confinement by the barriers.

Because the thickness of the strained layers is only on the order of several nanometers, the quantum effect should be taken into consideration. However, the generation of NAW can be modeled by a continuum elastic theory [8], [9], which is valid in the coherent regime as the thermal and quantum fluctuations can be neglected, as is in our cases. This macroscopic approach is similar to the theory 
of the typical piezoelectric transducer model. The macroscopic model is based on the constitutive equations taking into account both the piezoelectric and deformation couplings. The governing dynamic equations are the elastic wave equations coupled to the Poisson equation. Because the acoustic velocities of $\mathrm{GaN}$ and $\mathrm{InN}$ are close to each other and the In composition of InGaN is low, we can neglect the discontinuities of elastic and dielectric properties between wells and barriers and treat the InGaN/GaN MQW as a continuum elastic body.

Here we use the wurtzite crystalline structure as an example, and the general principle also can be applied to other crystalline systems. We use $(x, y, z)$ coordinate system to denote the primary crystallographic axes of the wurtzite based nitride system. The $z$-direction is chosen to be along the crystal $c$-axis and $\hat{\mathbf{N}}$ denotes the crystal growth direction. We start from the system-free energy density $F[15]-[17]$ that can be described as:

$$
\begin{array}{r}
F(T, \mathbf{E}, \boldsymbol{\epsilon})=F_{0}(T)+\frac{1}{2} C_{i j k l}^{(T)} \boldsymbol{\epsilon}_{i j} \boldsymbol{\epsilon}_{k l}-\frac{1}{2} \varepsilon_{i j}^{(\boldsymbol{\epsilon})} E_{i} E_{j}, \\
-e_{i j k} E_{i} \boldsymbol{\epsilon}_{j k}+\boldsymbol{\epsilon}_{i i} d_{v i} \rho_{v}
\end{array}
$$

where we have taken temperature $T$, electric field $\mathbf{E}=$ $\left(E_{x}, E_{y}, E_{z}\right)$, and strain $\epsilon$ as independent thermodynamic variables. In the above expression, $F_{0}, C_{i j k l}^{(T)}, \varepsilon_{i j}^{(\boldsymbol{\epsilon})}$, and $e_{i j k}$ are the free energy per unit volume before deformation, isothermal elastic stiffness tensor, dielectric tensor, and piezoelectric tensor, respectively. The index $v$ runs over carrier species such as electrons, heavy holes, light holes, etc. $\rho_{v}$ is the density of carrier species $v$, and $d_{v i}$ is the corresponding deformation potential tensor. The last two terms in (1) represent piezoelectric and deformation couplings, respectively. From the Maxwell's equation, constitution law, and the equation of motion, the loaded wave equation can be derived as [8], [9]:

$$
\rho_{0} \frac{\partial^{2} \mathbf{u}}{\partial t^{2}}-\bar{\Gamma} \cdot \frac{\partial^{2} \mathbf{u}}{\partial z^{\prime 2}}=\mathbf{f}_{\text {piezo }}+\mathbf{f}_{\text {def }},
$$

where $\rho_{0}$ is the mass density, $\mathbf{u}$ is the displacement vector field, and $z^{\prime}$ axis is along the growth direction of the strained layers. $\bar{\Gamma}$ is the effective Christoffel tensor with elements:

$$
\bar{\Gamma}_{i l}=\Gamma_{i l}+\frac{\bar{e}_{i} \bar{e}_{l}}{\bar{\varepsilon}}
$$

and

$$
\Gamma_{i l}=C_{i j k l}^{(T)} N_{j} N_{k}
$$

is the second-rank Christoffel tensor [16]. $\bar{\varepsilon}$ is the effective dielectric constant, and $\bar{e}_{i}$ is the effective piezoelectric tensor. $N_{j}$ denotes a basis vector in the coordinate, in which one basis vector is along $\hat{\mathbf{N}}$ and the other two are orthogonal with each other on the plane normal to $\hat{\mathbf{N}}$. In (2), the two driving force fields are:

$$
\mathbf{f}_{\text {piezo }}=-\frac{\overline{\mathbf{e}}}{\bar{\varepsilon}} \rho_{\mathbf{s c}}=|e| \frac{\overline{\mathbf{e}}}{\bar{\varepsilon}}\left(\rho_{\text {elec }}-\rho_{\text {hole }}\right),
$$

and

$$
\mathbf{f}_{\mathbf{d e f}}=\sum_{v} \overline{\mathbf{d}}_{v} \frac{\partial \rho_{v}}{\partial z^{\prime}}
$$

where $\overline{\mathbf{d}}_{v}$ is the deformation tensor of carrier species $v$.

Expression (2) describes the wave dynamics of the displacement vector field propagating along the growth direction of the strained layers. On the right-hand side, two driving force terms determine the initiated waveform. The first one is the piezoelectric force due to the generation of photocarriers in the wells as depicted in (5). The second one is the deformation coupling due to the nonhomogeneous distribution of photocarriers as represented in (6). As described in (2), the photocarriers play an important role to initiate NAW. It is of note that the strain pulses also can be initiated by thermal expansion. However, the relatively slower thermal stress generation process can be neglected for the generation of terahertz NAW, not to mention that thermal stress is much weaker compared with deformation stress in a semiconductor [18]. Moreover, a previous theoretical calculation revealed that the piezoelectric stress is $>5$ times the deformation stress [7], which supports the fact that the driving force in OPT is dominated by the piezoelectric force term [6]-[9].

Because optical velocity is far faster than acoustic velocity, the driving force can be treated as a step function. The fs-photoexcited carriers instantaneously screen the background piezoelectric field in the wells and change the equilibrium state of strain. By designing periodic well distributions, we thus can create a periodic strain variation pattern, and the wavelength of thus launched NAW then is determined by the periodicity of the strain variation (quantum well) pattern.

In our case, InGaN and GaN strained layers with several nanometer thickness form wells and barriers, respectively. The direction of growth $\hat{\mathbf{N}}$, which also means the direction of the driving force, is along the c-axis, and only longitudinal acoustic (LA) mode is excited. The equations are thus simplified to LA waves in one dimension. From (2), the strain waves are initiated and travel in two counterpropagation directions along the c-axis.

\section{Optical Detection of Nano-Acoustic Waves}

When the NAW propagates in a piezoelectric material, it results in modulation of the piezoelectric field (due to strain modulation in the piezoelectric material) and then causes optical absorption variation through the quantumconfined Franz-Keldysh (QCFK) effect [4]-[9], thus allowing us to optically detect the generated NAW using thin piezoelectric layers. In our case, we use the same OPT as the detecting structure, which has a periodic strained-layer structure with a spatial periodicity equivalent to the detected acoustic wavelength. The optical transmission vari- 


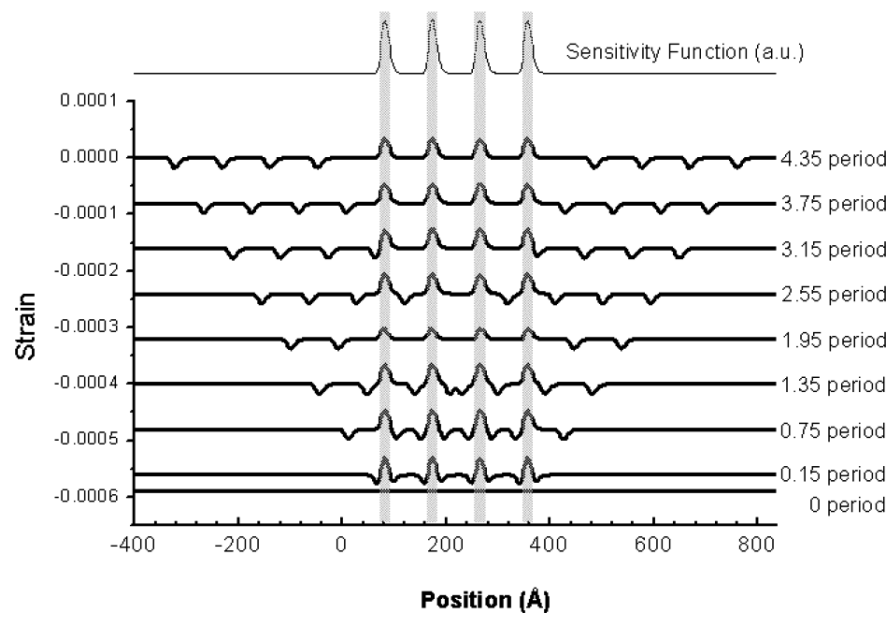

Fig. 2. Calculated strain fields at consecutive time delays (with a spacing equal to 0.6 period). Also shown is the calculated sensitivity function for a four-period optical piezoelectric transducer.

ation in the OPT due to the presence of strain waves can be derived as [19]:

$$
\left(\frac{\Delta T(t)}{T}\right)_{\mathbf{L A}}=\int_{-\infty}^{\infty} d z s(z, t) \cdot F(z ; \omega)
$$

where $s(z, t)=\partial u(z, t) / \partial z$ is the strain function and $F(z ; \omega)$ is the sensitivity function (dominated by the QCFK effect), which can be theoretically calculated according to the structure of the OPT [19]. The sensitivity function represents the optical absorption modulation strength (or the optical transmission modulation strength) at a specific optical frequency due to the strain variation at a specific position. For example, we have calculated the sensitivity function of a four-period InGaN SLS (or MQW) OPT (top of Fig. 2). In our case, the photon energy of the optical probe is above the bandgap of the InGaN layer but below that of the GaN layer, and thus the nonzero value of the sensitivity function in the InGaN region means that transmission of optical probe beam is strongly modulated while the strain wave enters the InGaN strained layers. With a fs optical pulse much shorter than the acoustic period, the InGaN strained layers can be used to detect the NAW with resolved phase information. For example, we need a probe pulse shorter than 250 fs to optically detect a $1 \mathrm{THz}$ acoustic wave. The optical frequency of the probe should be selected based on the $F(z ; \omega)$ so that a high sensitivity can be achieved.

Fig. 2 also shows the simulated time evolution of the spatial strain distribution variation. When the OPT is excited by a fs optical pulse, the strain distribution is immediately altered from its original equilibrium and two counter-propagating NAWs are initiated. From (7), propagation of the NAWs in the OPT can be monitored through the periodic sensitivity function of the MQW by recording the transient transmission modulation $(\Delta T / T)$ of an optical probe pulse. It is worthwhile to note that the modulated optical transmission signal is a correlation function between the strain wave and the sensitivity function as (a)

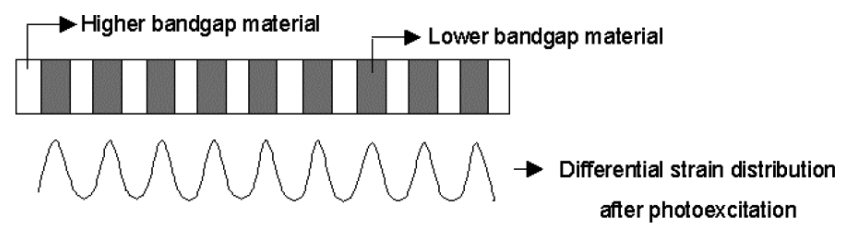

(b)

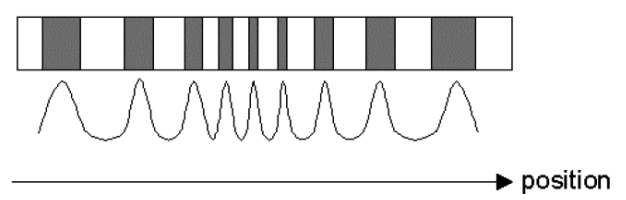

Fig. 3. Schematic band diagram of strained-layer-structured OPT to generate (a) fixed frequency and (b) dispersed NAW. The white and gray regions represent higher bandgap material and lower bandgap material, respectively. The differential strain distribution after photoexcitation is plotted below the multiple strained-layer-structure OPT.

represented in (7). The sensitivity function can be treated as a matched filter because its period width is the same as the wavelength of the NAW. With fs optical pulses, the periodic SLS OPT is not only a source to induce NAW with a wavelength determined by the period of strained layers, but also a matched filter to detect the NAW it generated.

\section{IMPUlse Response of the OPT}

The impulse-generated acoustic waveform in an OPT is determined by the photocarrier-induced variation of the electric field pattern, which is on the other hand determined by the pattern of the multiple SLS. For example, the period of piezoelectric InGaN/GaN MQW determines the generated acoustic wavelength, and the ratio of barrier width to well width in the same MQW determines its Fourier component composition [20]. Fig. 3 illustrates the conceptual principle to design a pattern for a specific impulse response waveform. A structure to generate a fixed wavelength acoustic wave is shown in Fig. 3(a). The gray region is the quantum well with lower bandgap, and the white region is the barrier with higher bandgap. The differential strain distribution induced by the fs photoexcited carriers is plotted below the structure diagram. This sudden difference of strain distribution results in acoustic waves as shown in Fig. 2. Because the carriers are generated in the wells, the period width of the nano-SLS determines the generated acoustic wavelength. It is of note that the strain waveform distribution is not a pure sinusoidal function but one composed of harmonic Fourier components. For a fixed-frequency acoustic wave, the waveform distribution can be designed simply by controlling the thickness ratio of the barrier layer and the well layer. Apart from a fixed-frequency acoustic wave, a chirped-frequency waveform also can be generated as schematically shown in Fig. 3(b). The intervals of strain oscillation can be designed by the intervals of adjacent wells. Conceptually, arbitrary waveforms can be generated through the design of the OPT nano-structure. Detailed waveform of the in- 


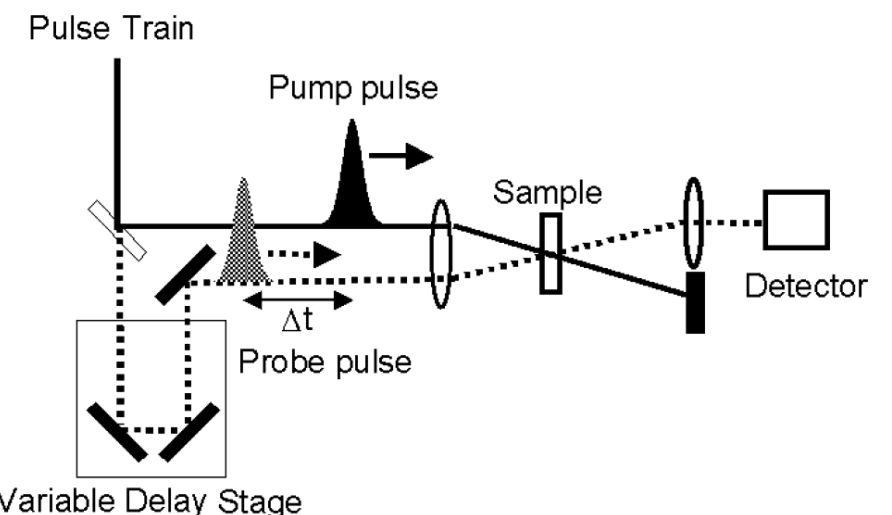

Fig. 4. The schematic of transmission fs pump-probe measurement. The fs-optical pulse is split into pump pulse (solid line) and probe pulse (dotted line). The time delay between the two pulses is controlled by a delay stage. After focusing the two pulses onto the sample, the transient transmission change of the probe pulse is recorded through an optical detector.
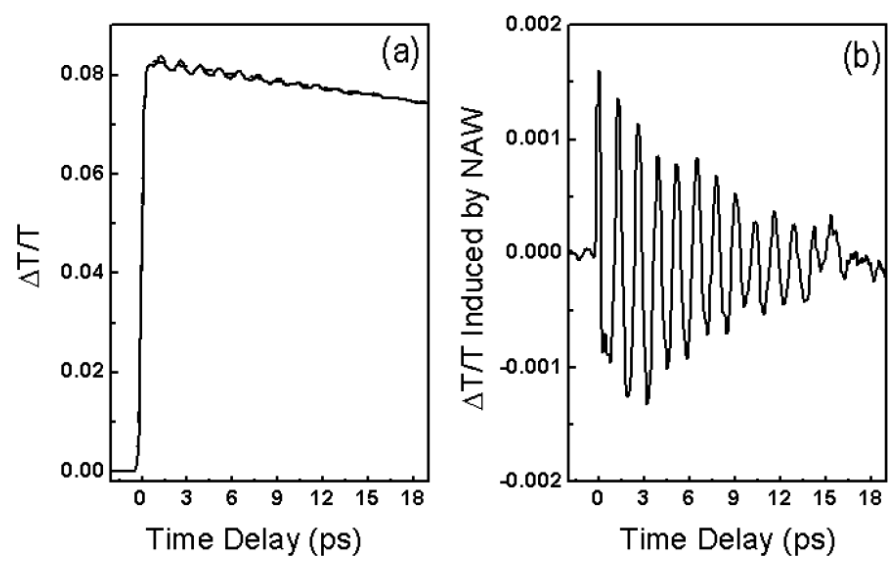

Fig. 5. (a) Measured optical transient transmission change versus probe delay for a 14-period $22 \AA / 70 \AA$ InGaN/GaN MQW OPT (solid line). The optical central wavelength is $\sim 390 \mathrm{~nm}$. The fitted background signal is shown as a dashed line. (b) Measured transient transmission change versus optical probe delay after removing the background signal.

duced NAW can be mathematically calculated according to $[6]-[9]$.

\section{Experimental Characterization of the OPT}

To demonstrate the generation and detection of NAW, we have used a standard optical transmission pump-probe system [21] as shown in Fig. 4. The optical pump pulse is for inducing the NAW, and the probe pulse is for monitoring the propagation of the NAW. Transient measurement is achieved by scanning the time (spatial) delay between the pump and probe pulses. Fig. 5(a) shows the typical probe transmission changes as a function of pump-probe time delay. The optical pulsewidth is $\sim 250 \mathrm{fs}$, and the central wavelength of the pump and probe pulses is $390 \mathrm{~nm}$. At this wavelength, the optical probe has high sensitivity due to the QCFK effect [5], [19]. The average pump power is $\sim 28 \mathrm{~mW}$, and the diameter of the optical spot size on the sample is $\sim 14 \mu \mathrm{m}$. The pump-induced, 2-D carrier density in one InGaN well is estimated to be $8 \times 10^{12} \mathrm{~cm}^{-2}$. When the pump excites carriers in sample A, transient transmission increases with zero time delay due to a sudden population increase, and a clear cosine-like transmission oscillation (due to the pump-induced NAW) can be observed on top of the background carrier dynamics signal [22]. We have subtracted the background signal with a fitting procedure [23], and the NAW-induced optical transmission (T) modulation can be extracted as shown in Fig. 5(b). Large transmission modulation $\Delta T / T$ on the order of or higher than $10^{-3}$ can be observed even with a relatively weak optical excitation (with $3.6 \times 10^{-4} \mathrm{~J} / \mathrm{cm}^{2}$ optical fluence). The typical noise background in our optical system is about $10^{-5}-10^{-6}$ [24]. Further reduction of noise is possible.

Because of the ultrashort optical pump pulsewidth, the experimental trace in Fig. 5(b) can be treated as the impulse response of the nano-structured OPT in sample A. The NAW-modulated $\Delta T / T$ depth decreases with evolution of time, reflecting that the two counter-propagating NAWs are traveling away from the OPT in the direction perpendicular to the strained-layer plain, as discussed in Section IV. Because there are 14 strained-well layers in sample A, 14 oscillation cycles are expected before the NAW completely leaves the OPT. The observed acoustic oscillation in sample A $(22 \AA / 70 \AA \mathrm{InGaN} / \mathrm{GaN} \mathrm{MQW}$ with $9.2 \mathrm{~nm}$ acoustic wavelength) is $\sim 1.25 \mathrm{ps}$, corresponding to an acoustic frequency of $0.8 \mathrm{THz}$. Fourier analysis reveals that higher harmonic components are observed due to its highly asymmetric well-barrier-width ratio ( 2.2 versus 7.0). The detailed spectral analysis of an OPT has been discussed elsewhere [20].

\section{Transient Waveform Synthesis}

The nano-structure of an OPT determines the impulsegenerated acoustic waveform that is initiated by a fs optical pulse. Thus, it is possible to further manipulate the waveform by optical engineering to design the transient driving force (usually called optical coherent control). We previously demonstrated the optical coherent control of acoustic phonon oscillations. The manipulation, including magnitude and phase, of the coherent acoustic phonon oscillations has been demonstrated [21], [25] through optical engineering of a fs pulse sequence. This coherent control technique thus can be applied to manipulate the magnitudes and phases of different embedded frequency components of the OPT, thus achieving the nano-acoustic waveform synthesis.

Here we take a fixed-frequency sample (sample A) as an example to demonstrate the manipulation of its different acoustic Fourier components. The fundamental frequency of sample $\mathrm{A}$ is $\sim 0.8 \mathrm{THz}$, and higher harmonic components also are observed [20]. By injecting an amplitudecontrolled fs control pulse at a 2.5 oscillation cycle time 


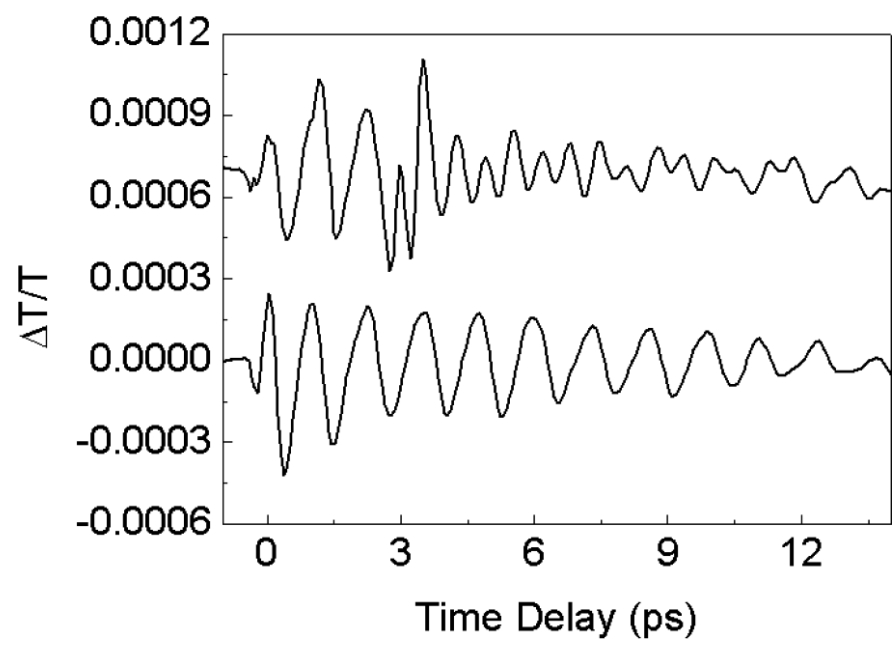

Fig. 6. Optically generated NAW in a 14-period $22 \AA / 70 \AA$ In$\mathrm{GaN} / \mathrm{GaN}$ OPT. The background signals are subtracted. Bottom trace shows the detection of NAW with $9.2 \mathrm{~nm}$ wavelength $(0.8 \mathrm{THz})$. Top trace demonstrates our developed coherent control technique that alters the acoustic wavelength to $4.6 \mathrm{~nm}(1.6 \mathrm{THz})$ after $3.1 \mathrm{ps}$.

delay, we successfully cancel the $0.8 \mathrm{THz}$ acoustic component but enhance the $1.6 \mathrm{THz}$ acoustic component. This transient trace of the coherent control experiment is shown in Fig. 6, and the original impulse response also is shown for comparison. The NAW with $1.6 \mathrm{THz}$ frequency is transiently amplified after injecting the control pulse at a $3.1 \mathrm{ps}$ time delay.

Conceptually, every fs optical pulse in a well-controlled pulse sequence initiates a NAW with a specific intensity, and the time intervals between different fs optical pulses determine the phase (and time) delay between different NAWs. Superposition of every NAW with specific intensities and phases provides a way to design and control a variety of different waveforms. Transient waveform synthesis of NAW provides not only a source for nano-ultrasonics but also a resource for many acoustics-related researches such as the transient elastic property studies with fs and nanometer resolutions.

\section{One-Dimensional Scan Measurement}

With the acoustic generation and detection technique provided by OPT, we easily can relate it to the application of 1-D ultrasonic-scan measurement. Because the nanoSLS OPT generates acoustic waves with nanometer wavelengths, the spatial resolution of the 1-D scan is thus also on the scale of nanometer. In Fig. 5(b), we observed the acoustic signal with 14 oscillation cycles resulting from the process of NAW propagating outward from the OPT. However, an acoustic pulse with too many oscillation cycles will seriously affect its resolution to determine the position of the echoed signal. To design an OPT for 1-D ultrasonic scan measurement, NAW with fewer oscillation cycles (i.e., with a shorter acoustic pulsewidth) is required. However, according to the sensitivity function, fewer period-strained

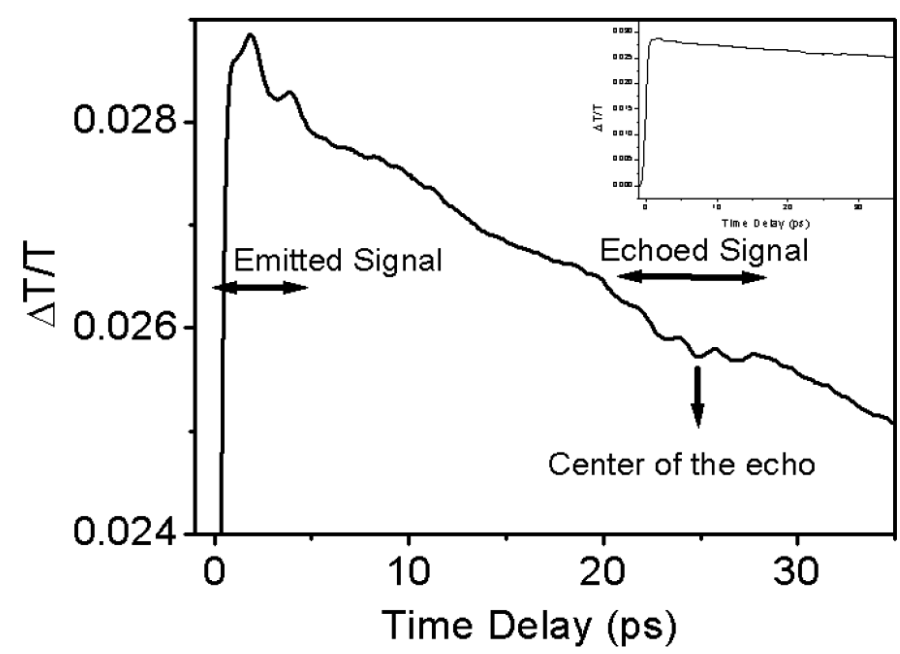

Fig. 7. Measured optical transient transmission changes versus probe delay for a three-period $29 \AA / 130 \AA$ InGaN/GaN OPT. The complete trace is shown in the inset.

layers will decrease the amplitude of the detected optical signal and increase the detection bandwidth. But this situation can be improved with a higher signal-to-noise ratio (SNR) optical detection system.

To improve the SNR in our optical detection system, we have replaced the mechanical chopper (providing $\sim 900 \mathrm{~Hz}$ chopping frequency) [21] with an acousto-optical modulator for a higher chopping frequency $(\sim 500 \mathrm{kHz})$ and lower system noise. The 1-D ultrasonic scan measurement was performed in sample $\mathrm{B}$, in which the three-period $29 \AA / 130 \AA \mathrm{In}_{0.23} \mathrm{Ga}_{0.77} \mathrm{~N} / \mathrm{GaN}$ MQW region acts as the OPT. On top of the InGaN/GaN MQW, the sample is capped with a GaN layer for the thickness measurement. A rough estimate on the GaN cap-layer thickness $(\sim 80 \mathrm{~nm})$ and the buffer-layer thickness $(\sim 3.4 \mu \mathrm{m})$ can be obtained according to the growth rate.

Fig. 7 shows the result of the transient optical transmission measurement for the three-period $29 \AA / 130 \AA$ InGaN/GaN SLS OPT (sample B). The optical modulation induced by the NAW propagating inside the OPT structure is clearly observable in the first 5 ps and around $25 \mathrm{ps}$. The oscillating period is $2 \mathrm{ps}$, corresponding to an acoustic frequency of $0.5 \mathrm{THz}$. Around zero time delay when the strain pulse is generated in the quantum-well region of the OPT, an increase in the optical transmission is expected but is not resolved in our optical signal due to the finite optical pulsewidth [5]. Although the periodic strain pulses traveling out of the wells where they are generated, two transmission oscillation peaks can be observed after zero time delay. One generated NAW leaves the OPT and travels toward the surface following the sample growth direction, is reflected at the sample surface, and echoes back into the OPT starting at $\sim 20 \mathrm{ps}$. It is valuable to note that the echoed strain pulse decreases the background optical transmission because the sign of the echoed strain is reversed at the GaN/air interface, which is a free end. Traveling through the MQW region, the echoed NAW with three cycles results in five optical transmission dips on the 


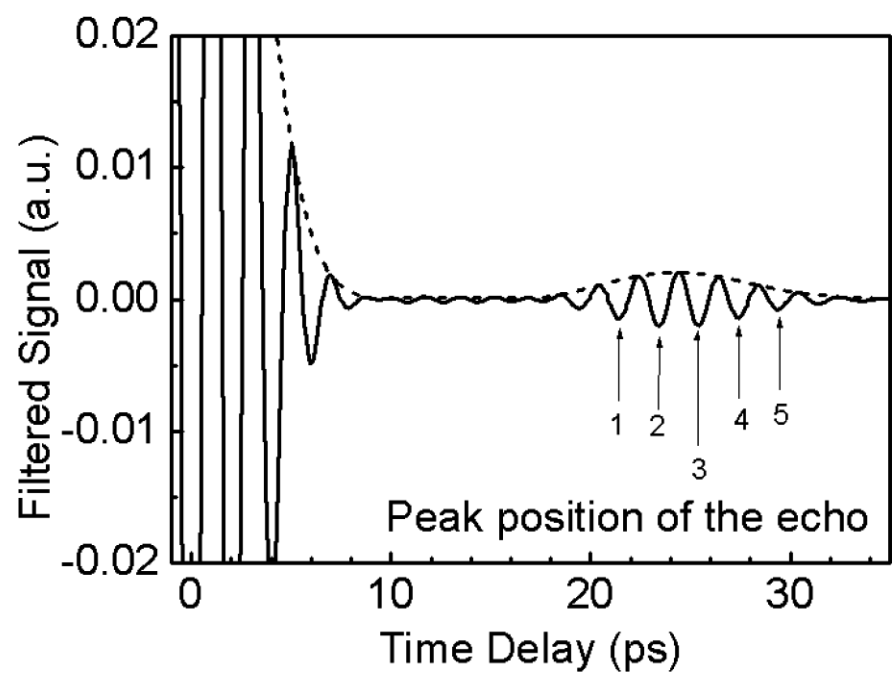

Fig. 8. The trace in Fig. 7 after filtering (solid line). Its envelope is shown as a dashed line. Five echoed peaks are clearly observable. Two more side peaks are caused by filter artifact.

background signal. The transient central position of the five dips corresponds to the moment when the three-cycle NAW is located in the three quantum wells. It is of note that, due to the nonflat background signal, the peak position time could be shifted. The fitting process used in Fig. 5 provides a method to obtain the acoustic signals and the exact echo position without losing the waveform information. For 1-D thickness measurement, the most important information is the peak position time in the echoed signals. Because the spectrum of the acoustic signals can be extracted, the measured traces can be processed by a bandpass filter.

To filter the background signal and accurately resolve the peak position of the echo signal, the optical trace has been processed as follows. The echo signal $x(t)$ has been digitized with a sampling frequency $f_{s}$ of $15 \mathrm{THz}$ into $x[n]$, that is:

$$
x[n]=x\left(n / f_{s}\right) .
$$

Fig. 7 shows the echo signal $x[n]$. A Gaussian-shaped, bandpass filter then was applied on $x[n]$ to remove the out-of-band noise. The filter is:

$$
H_{\mathbf{G}}[n]=\exp \left[-A\left(\frac{n}{f_{\mathbf{S}}}\right)^{2}\right] \cdot \sin \left(2 \pi f_{\mathbf{c}} \frac{n}{f_{\mathbf{S}}}\right),
$$

where $f_{\mathbf{c}}=0.5 \mathrm{THz}$ is the center frequency of the echo signal and $A=\frac{\pi^{2}\left(f_{\mathrm{c}} / 3\right)^{2}}{\ln 40}$. Note that the value of $A$ has been chosen to make the $-40 \mathrm{~dB}$ bandwidth of $H_{\mathbf{G}}[n]$ match the bandwidth (defined as the width between two zero-crossing points around the center frequency) of the expected echo signal, which is $2 f_{\mathbf{c}} / 3$. The filtered signal $x_{\mathbf{F}}[n]=x[n] \otimes h_{\mathbf{G}}[n]$, where $\otimes$ represents the convolution operation, is shown in Fig. 8. The arrival time of the echo can be found from the envelope of $x_{\mathbf{F}}[n]$ :

$$
x_{\mathbf{E}}[n]=\left|\mathbf{H}\left\{x_{\mathbf{F}}[n]\right\}\right|,
$$

where $\mathbf{H}$ denotes the Hilbert transform [26]. By finding the peak position of $x_{\mathbf{E}}[n]$, we have found that the peak position of the echo signal (the third oscillation peak) is about 25.4 ps with respect to the excitation time.

It should be noted that the peak position of 25.4 ps does not represent the traveling time of NAW during the GaN cap layer. It represents the required time that the generated NAW travels into the cap layer, is reflected from the cap/air interface, then goes back to match the period of strained layers in OPT. To calculate the accurate thickness of the GaN cap layer grown on the OPT, the time that NAW travels in the cap layer should be obtained by subtracting the time spent in the OPT. In the case of three-period SLS OPT, three strain pulses are generated in the three InGaN well layers. We take the strain pulse generated in the first InGaN strained layer below the GaN cap layer (called strain pulse A) as an example to calculate the traveling time in the GaN cap layer. When the strain pulse A comes back from the GaN/air interface, it enters the first InGaN strained layer below the GaN cap layer and results in the first dip signal of the echo signal as shown in Fig. 7. When the strain pulse A crossing the second well arrives the third well, it results in the third dip of the echo signal, which is the peak position. Therefore, the traveling time in the GaN cap layer is that of the echo peak position subtracting the time in the OPT with two GaN barriers and three InGaN wells. With an oscillation period of $2 \mathrm{ps,}$ the traveling time in the OPT is $4.4 \mathrm{ps}$, resulting in $21.0 \mathrm{ps}$ traveling time in the GaN cap layer. Assuming the sound velocity of GaN along c-axis is $8020 \mathrm{~m} / \mathrm{s}$ [27], the thickness of the GaN cap layer is thus estimated to be $\sim 84 \mathrm{~nm}$.

For a single-layer thickness measurement without multiple reflections, taking advantage of the phase information, we can achieve a resolution better than $20^{\circ}(\lambda / 16)$, corresponding to $<1 \mathrm{~nm}$ resolution in this specific case. However, the uncertainty of the acoustic velocity also results in measurement errors. For example, a $200 \mathrm{~m} / \mathrm{s}$ uncertainty [11] gives rise to $2.5 \%$ inaccuracy for the determination of the cap layer thickness (in this case, $2.1 \mathrm{~nm}$ ). However, the acoustic velocity also can be accurately obtained by comparison of different thickness cap layer on the same OPT.

\section{Discussion}

The technique of generating and detecting NAW in nano-SLS OPT has several advantages:

- High speed. In a typical electronic piezoelectric transducer, the generation and detection systems usually operate through the same electrode, and a switch is required. However, for a nano-structured OPT, the system is operated by two independent optical pulses. The interference time of both optical pulses may be treated as the effective switching time, which is determined by the optical pulsewidth. The effective switch time thus can be very short, for instance 
only limited by the shortest 4 fs optical pulse generated from a state-of-the-art Ti:sapphire laser [28]. In most commercial Ti:sapphire laser systems, an optical pulsewidth less than 100 fs can be achieved easily.

- High spatial resolution. Because the time resolution of the optical detection is determined by the optical probe pulse width, not only high speed but also high spatial resolution thus can be achieved. For example, with an optical probe-pulsewidth of $100 \mathrm{fs}$ and an acoustic velocity of $8000 \mathrm{~m} / \mathrm{s}$, the spatial resolution is better than $0.8 \mathrm{~nm}$ (with a fitting procedure, a time resolution better than pulsewidth can be achieved easily as in our case). As for the detection of thin multilayers, the actual spatial resolution also is restricted by the acoustic wavelength and the acoustic pulsewidth. By resolving the envelope of the reflected acoustic echo, NAW easily can provide a spatial resolution down to several nanometers. By resolving the phase information and the interference between different echoes due to multilayers, a spatial resolution down to several angstroms is possible.

- Optically determined acoustic beam size. The acoustic beam size of the demonstrated technology is determined by the spot size of the excitation optical pulses. The cross-section area of the OPT thus can be tuned by controlling the illuminating area. In our case, the diameter of the focused optical spot size is about $10 \mu \mathrm{m}$, and the generated NAW should be treated as plane waves. To further reduce the spot size, high numerical-aperture objective lens can achieve $<200 \mathrm{~nm}$ diameter focused spot-size at an ultraviolet optical wavelength. At the same time, NAW can be generated at arbitrary positions on the plane normal to the growth direction, controlled by the optical focal positions. This could be a powerful tool for acoustic wave source engineering. For example, we can achieve lateral scanning without physically separated piezoelectric transducer arrays.

Compared with the acoustic wave source provided by the optical technique of picosecond ultrasonics with picosecond resolution, the NAW generated from an OPT with well-controlled oscillation cycles and waveform can have the following advantages:

- Pulseshape and oscillation cycle engineering capability. The number of oscillation cycles is determined by the number of strained layers. However, the material and width of alternating strained layers determine the pulseshape. The demanded shape and oscillation cycles of NAW thus can be achieved by designing the nano-structure of an OPT.

- Phase engineering capability. Phase information is crucial in 3-D ultrasonic imaging processing. The ability to design the waveform and phase of the acoustic source with nanometer wavelength also provides a powerful tool for many studies such as elastic property study in the regime of nano-scale and terahertz response.
- Better SNR. Because the frequency, oscillation cycles, and waveform of NAW can be preprogrammed, optical and electronic noises can be removed easily with signal-processing techniques.

In Section VIII, we demonstrated how an OPT was applied to a phase-sensitive 1-D ultrasonic scan, which is the first step toward 3-D nano-ultrasonic imaging. However, to obtain nano-ultrasonic imaging, the acoustic beam size determined by the excitation area of fs pulses also should be on the nanometer scale. It can be done by using near-field optical techniques. Besides, typical ultrasonic imaging is composed of back-scattered signals. The scattered signal also may provide the information of the crystal quality in a nanometer scale, e.g., defect distributions in the semiconductor samples. To detect the scattered signals, larger amplitude NAW and higher SNR of the optical system are required. It is possible to study the scattered signals based on our previous demonstrated system because the excitation pulse energy of $0.2-0.4 \mathrm{~nJ}$ in the experimental optical system here is still low, ${ }^{1}$ and the SNR can be raised two orders if fast scan technique [29] could be incorporated. Our demonstration reveals that the OPT provides the key technology toward the realization of nano-ultrasonics.

\section{Conclusions}

Nano-strained-layer structures in piezoelectric semiconductors can be treated as an optical piezoelectric transducer. With fs optical pulses, photoexcited carriers result in the periodic piezoelectric and deformation driving forces, and thus initiate nano-acoustic waves. The wavelength of the induced-NAW is determined by the period width of the alternating strained layers and easily can be less than $10 \mathrm{~nm}$. The impulse-generated NAW waveform can be calculated theoretically according to the structure of the OPT. Through the QCFK effect, the propagating NAW in the OPT structure will modulate its optical property, and thus its transient behavior can be monitored by the other optical probe pulse. Because the generation and detection positions of the NAW are determined by the illuminating areas, it is easy to select the active region by tuning the spot size and the excitation position of the optical pulses. The simultaneous manipulation of the phase and magnitude of different acoustic-frequency components could be achieved by optical coherent control, thus accomplishing nano-acoustic waveform synthesis. We also have demonstrated 1-D nano-ultrasonic scans with the OPT. Under our experimental conditions, a spatial resolution on the order or better than $1 \mathrm{~nm}$ can be achieved. The ultrafast optical technique and the phase-sensitive OPT allow the realization of nano-ultrasonics. With the techniques of waveform engineering, high SNR optical systems, and nano-acoustic devices, nano-ultrasonic imaging has the potential to be realized in the near future.

\footnotetext{
${ }^{1} \mathrm{~A}$ pulse energy of frequency-double Ti:sapphire optical pulse up to $10 \mathrm{~nJ}$ is achievable.
} 


\section{ACKNOWLEDGMENT}

Sample A was kindly provided by S. Keller and S. P. DenBaars of the University of California at Santa Barbara. K.-H. Lin would like to thank SiS Education Foundation for financial support.

\section{REFERENCES}

[1] H. T. Grahn, H. J. Maris, and J. Tauc, "Picosecond ultrasonics," IEEE J. Quantum Electron., vol. 25, pp. 2562-2569, 1989.

[2] H. Maris, "Picosecond ultrasonics," Sci. Amer., vol. 278, pp. 86-89, 1998.

[3] A. Devos and C. Lerouge, "Evidence of laser-wavelength effect in picosecond ultrasonics: Possible connection with interband transitions," Phys. Rev. Lett., vol. 86, pp. 2669-2672, 2001.

[4] C.-K. Sun, J.-C. Liang, A. Abbar, L. Coldren, and S. P. DenBaars, "Large coherent acoustic phonon oscillation observed in InGaN/GaN multiple-quantum-wells," Appl. Phys. Lett., vol. 75, pp. 1249-1251, 1999.

[5] C.-K. Sun, J.-C. Liang, and X.-Y. Yu, "Coherent acoustic phonon oscillations in semiconductor multiple-quantum-wells with piezoelectric fields," Phys. Rev. Lett., vol. 84, pp. 179-182, 2000 .

[6] G. D. Sanders, C. J. Stanton, and C. S. Kim, "Theory of coherent acoustic phonons in $\operatorname{In}_{x} \mathrm{Ga}_{1-x} \mathrm{~N} / \mathrm{GaN}$ multiple quantum wells," Phys. Rev. B, vol. 64, pp. 235316-1-235316-18, 2001.

[7] G. D. Sanders, C. J. Stanton, and C. S. Kim, "Erratum: Theory of coherent acoustic phonons in $\operatorname{In}_{\mathrm{x}} \mathrm{Ga}_{1-\mathrm{x}} \mathrm{N} / \mathrm{GaN}$ multiple quantum wells," Phys. Rev. B, vol. 64, pp. 079903-1-079903-3, 2001.

[8] G.-W. Chern, C.-K. Sun, Y.-K. Huang, and K.-H. Lin, "Generation of coherent acoustic phonons in piezoelectric semiconductor heterostructures," Proc. SPIE, vol. 4992, pp. 226-240, 2003.

[9] G.-W. Chern, C.-K. Sun, G. D. Sanders, and C. J. Stanton, "Generation of coherent acoustic phonons in nitride-based semiconductor nanostructures," Top. Appl. Phys., vol. 92, pp. 339$390,2004$.

[10] S. Nakamura and G. Fasol, The Blue Laser Diode. New York: Springer, 1997

[11] R. J. Jiménez Riobóo, E. Rodríguez-Cañas, M. Vila, C. Prieto, F. Calle, T. Palacios, M. A. Sánchez, F. Omnès, O. Ambacher, B. Assouar, and O. Elmazria, "Hypersonic characterization of sound propagation velocity in $\mathrm{Al}_{\mathrm{x}} \mathrm{Ga}_{1-\mathrm{x}} \mathrm{N}$ thin films," J. Appl. Phys., vol. 92, pp. 6868-6874, 2002.

[12] S. F. Chichibu, A. C. Abare, M. S. Minsky, S. Keller, S. B. Fleischer, J. E. Bowers, E. Hu, U. K. Mishra, L. A. Coldren, S. P. DenBaars, and T. Sota, "Effective band gap inhomogeneity and piezoelectric field in InGaN/GaN multiquantum well structures," Appl. Phys. Lett., vol. 73, pp. 2006-2008, 1998.

[13] C.-M. Lee, C.-C. Chuo, I.-L. Chen, J.-C. Chang, and J.-I. Chyi, "High-brightness inverted InGaN/GaN multiple-quantum-well light-emitting," IEEE Electron. Dev. Lett., vol. 24, pp. 156-158, 2003.

[14] C. Y. Lai, T. M. Hsu, W.-H. Chang, K.-U. Tseng, C.-M. Lee, C.-C. Chuo, and J.-I. Chyi, "Direct measurement of piezoelectric field in $\mathrm{In}_{0.23} \mathrm{Ga}_{0.77} \mathrm{~N} / \mathrm{GaN}$ multiple quantum wells by electrotransmission spectroscopy," J. Appl. Phys., vol. 91, pp. 531-533, 2002.

[15] L. D. Landau, E. M. Lifshitz, and L. P. Pitaevskii, Electrodynamics of Continuous Media. New York: Pergamon, 1984.

[16] D. Royer and E. Dieulesaint, Elastic Waves in Solids. New York: Springer-Verlag, 2000

[17] N. V. Chigarev, D. Y. Parachuk, X. Y. Pan, and V. E. Gusev, "Coherent phonon emission in the supersonic expansion of photoexcited electron-hole plasma in Ge," Phys. Rev. B, vol. 61, pp. 15837-15840, 2000.

[18] O. B. Wright, B. Perrin, O. Matsuda, and V. E. Gusev, "Ultrafast carrier diffusion in gallium arsenide probed with picosecond acoustic pulses," Phys. Rev. B, vol. 64, pp. 081202-1(R)-0812024(R), 2002.
[19] G.-W. Chern, K.-H. Lin, and C.-K. Sun, "Transmission of light through quantum heterostructures modulated by coherent acoustic phonons," J. Appl. Phys., vol. 95, pp. 1114-1121, 2004.

[20] G.-W. Chern, K.-H. Lin, Y.-K. Huang, and C.-K. Sun, "Spectral analysis of high-harmonic coherent acoustic phonons in piezoelectric semiconductor multiple quantum wells," Phys. Rev. B, vol. 67, pp. 121303-1-121303-4, 2003.

[21] C.-K. Sun, Y.-K. Huang, J.-C. Liang, A. Abare, and S. P. DenBaars, "Coherent optical control of acoustic phonon oscillations in InGaN/GaN multiple-quantum-wells," Appl. Phys. Lett., vol. 78, pp. 1201-1203, 2001.

[22] C.-K. Sun, F. Vallée, S. Keller, J. E. Bowers, and S. P. DenBaars, "Femtosecond studies of carrier dynamics in InGaN," Appl. Phys. Lett., vol. 70, pp. 2004-2006, 1997.

[23] K.-H. Lin, G.-W. Chern, S.-W. Chu, C.-K. Sun, H. Xing, Y. Smorchkova, S. Keller, U. Mishra, and S. P. DenBaars, "Ultrashort hole capture time in Mg-doped thin film," Appl. Phys. Lett., vol. 81, pp. 3975-3977, 2002.

[24] C.-K. Sun, J.-C. Liang, X.-Y. Yu, S. Keller, U. Mishra, and S. P. DenBaars, "Studies of carrier dynamics in unintensionally doped gallium nitride bandtail states," Appl. Phys. Lett., vol. 78, pp. $2724-2726,2001$.

[25] C.-K. Sun, G.-W. Chern, K.-H. Lin, and Y.-K. Huang, "Observation of large acoustic gain in coherent acoustic phonon oscillators," Chin. J. Phys., vol. 41, pp. 643-651, 2003.

[26] A. V. Oppenheim, R. W. Schafer, and J. R. Buck, Discrete-Time Signal Processing. 2nd ed. Englewood Cliffs, NJ: Prentice-Hall, 1999.

[27] C. Deger, E. Born, H. Angerer, O. Ambacher, M. Stutzmann, J. Hornsteiner, E. Riha, and G. Fischerauer, "Sound velocity of $\mathrm{Al}_{\mathrm{x}} \mathrm{Ga}_{1-\mathrm{x}} \mathrm{N}$ thin films obtained by surface acoustic-wave measurements," Appl. Phys. Lett., vol. 72, pp. 2400-2402, 1998.

[28] U. Keller, "Recent developments in compact ultrafast lasers," Nature, vol. 424, pp. 831-838, 2003.

[29] G. C. Cho, W. Kütt, and H. Kurz, "Subpicosecond time-resolved coherent-phonon oscillations in GaAs," Phys. Rev. Lett., vol. 65, pp. 764-767, 1990.

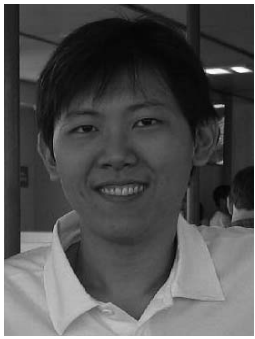

Kung-Hsuan Lin (S'03) was born in ChiaYi, Taiwan, in 1979. He received the B.S. degree in electrical engineering from National Taiwan University, Taipei, Taiwan, in 2001. He joined Ultrafast Optics (UFO) Group, Graduate Institute of Electro-Optical Engineering and Department of Electrical Engineering, National Taiwan University in 2001, and is currently working toward the Ph.D. degree.

His current research interests include nano-ultrasonics, and ultrafast phenomena in $\mathrm{GaN}$ and GaAs related semiconductors.

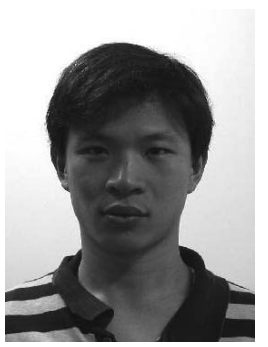

Gia-Wei Chern was born in Chia-Yi, Taiwan, in 1974. He received the B.S. degree in electrical engineering in 1996 and the Ph.D. degrees in Graduate Institute of ElectroOptical Engineering in 2001, from National Taiwan University, Taipei, R.O.C. In 2001, he joined the Ultrafast Optics Group, Department of Electrical Engineering and Graduate Institute of Electro-Optical Engineering, National Taiwan University, as a post doctor. He is currently working at the Department of versity, Baltimore, MD.
Physics and Astronomy, Johns Hopkins Uni- 


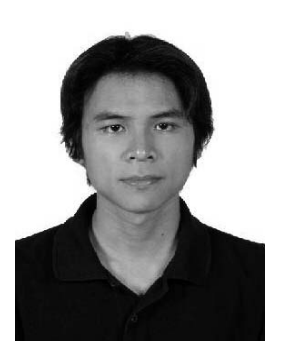

Cheng-Ta Yu was born in Taipei, Taiwan, in 1981. He received the B.S. degree in electrical engineering from National Taiwan University, Taipei, Taiwan, in 2003. He joined Ultrafast Optics (UFO) Group, Graduate Institute of Electro-Optical Engineering and Department of Electrical Engineering, National Taiwan University in 2003.

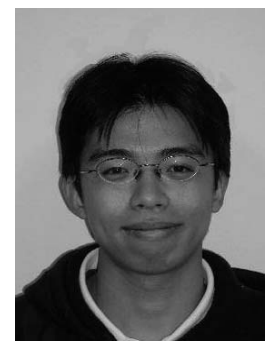

Tzu-Ming Liu was born in Keelung, Taiwan, R.O.C., in 1977. He received the B.S. degree in electrical engineering in 1999 from National Taiwan University, Taipei, R.O.C., where he is currently working toward the Ph.D. degree. In 1999, he joined the Ultrafast Optics (UFO) Group, Department of Electrical Engineering and Graduate Institute of Electro-Optical Engineering, National Taiwan University.

His current interests include carrier dynamics in semiconductor materials, ultrafast solid-state lasers, multiphoton confocal microscopy, and nonlinear optics.

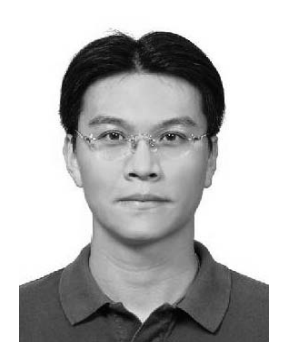

Chang-Chi Pan was born in Ping-Tong, Taiwan, R.O.C., in 1977. He received the M.S. degree from the Department of Electrical Engineering, Da-Yeh University, ChangHua, Taiwan, R.O.C., in 2001. He is currently working toward the Ph.D. degree at the Department of Electrical Engineering, National Central University, Chung-Li, Taiwan, R.O.C., where he is focusing his research on GaN-based optical devices growth and analysis.

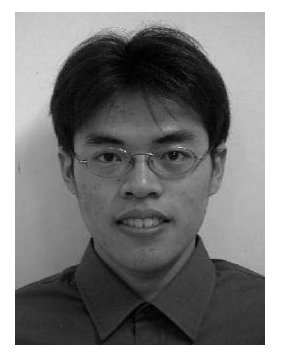

Guan-Ting Chen was born in Taiwan, R.O.C., in 1977. He received the B.S. and M.S. degrees in electrical engineering from National Central University, Chung-Li, Taiwan, in 1999 and 2001, respectively. He is currently working toward the $\mathrm{Ph} . \mathrm{D}$. program in electrical engineering, National Central University.

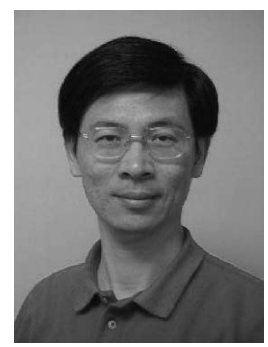

Jen-Inn Chyi received the B.S. and M.S. degrees in electrical engineering from $\mathrm{Na}$ tional Tsing-Hua University, Hsin-Chu, Taiwan, R.O.C., in 1982 and 1984, respectively, and the Ph.D. degree in electrical engineering from the University of Illinois, UrbanaChampaign, in 1990. His Ph.D. dissertation dealt with molecular beam epitaxial (MBE) growth and characterization of InSb on GaAs.

In 1991, he joined the Department of Electrical Engineering, National Central University, Jongli, Taiwan, as an associate professor. Since then, he has established MBE, metal-organic vapor phase epitxy (MOVPE), and high-speed optoelectronic devices laboratories, which house growth and characterization facilities for various
III-V materials and devices. He was appointed professor and Director of Optical Sciences Center in 1996 and 2000, respectively. His research interests are in the area of MBE and MOVPE growth of III-V semiconductors and their heterostructures for high-speed electronic and optoelectronic devices. His current research projects include MBE growth of InP-based heterojunction bipolar transistors, quantum dot photonic devices, MOVPE growth of GaN-based materials for ultraviolet, blue, green emitters and high temperature, high power devices.

Dr. Chyi has authored or co-authored over 200 journal papers, two book chapters, and has been granted seven patents. He is the recipient of the 1996 Distinguished Young Researcher Award, the 1996, 1998, and 1999 Best Paper Award of the Electronic Devices and Materials Association of R.O.C., and the 1996 and 1998 Engineering Paper Prize of the Chinese Institute of Engineers. He received the Distinguished Research Award of the National Science Council of R.O.C. in 2002. In 2004, he received the Distinguished Professor Award of the Chinese Institute of Electrical Engineering. He also serves as a Distinguished Lecturer of IEEE EDS since 2004. Phi

Dr. Chyi is a senior member of IEEE and a member of Phi Tau

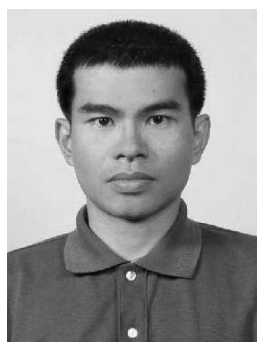

Sheng-Wen Huang was born in 1971 in Changhua, Taiwan, R.O.C. He received the B.S. and Ph.D. degrees in electrical engineering from National Taiwan University, Taipei, Taiwan, R.O.C., in 1993 and 2004, respectively. He is currently a postdoctoral researcher with the Department of Electrical Engineering, National Taiwan University.

His current research interests include ultrasonic tomography, coded excitation, and optoacoustic imaging.

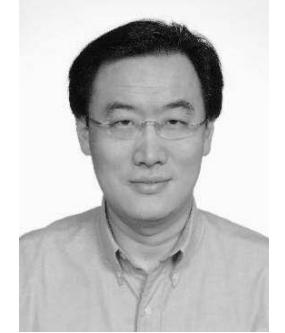

Pai-Chi Li (S'93-M'95-SM'01) received the B.S. degree in electrical engineering from National Taiwan University, Taipei, Taiwan, R.O.C., in 1987, and the M.S. and Ph.D. degrees from the University of Michigan, Ann Arbor, in 1990 and 1994, respectively, both in electrical engineering: systems.

He was a research assistant with the Department of Electrical Engineering and Computer Science of the University of Michigan from 1990 to 1994. He joined Acuson Corporation, Mountain View, CA, as a member of the technical staff in June 1994. His work in Acuson was primarily in the areas of medical ultrasonic imaging system design for both cardiology and general imaging applications. In August 1997, he went back to the Department of Electrical Engineering at National Taiwan University, where he is currently a full professor. His current research interests include biomedical ultrasonic imaging and signal processing.

Dr. $\mathrm{Li}$ is a senior member of IEEE and Associate Editor of IEEE Transactions on Ultrasonics, Ferroelectrics, and Frequency Control. He received the 2004 Distinguished Research Award, National Science Council, the 2004 Distinguished Research Achievement Award, National Taiwan University, the 2003 Outstanding Researcher Award, National Taiwan University, the 2002 Dr. Wu Dayou Research Award from National Science Council, the 2002 Outstanding Young Electrical Engineer Award from Chinese Institute of Electrical Engineering and the Distinguished Industrial Collaboration Award of Ministry of Education. He also was the recipient of the Distinguished Achievement Award in Electrical Engineering: Systems in 1994 for his outstanding academic achievement at the University of Michigan. 


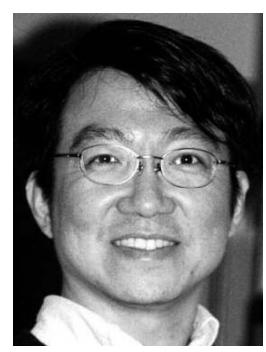

Chi-Kuang Sun (M'96-SM'01) was born in Tainan, Taiwan, R.O.C., in 1965. He received the B.S. degree in electrical engineering from National Taiwan University, Taipei, R.O.C., in 1987 and the M.S. and Ph.D. degrees in applied physics from Harvard University, Cambridge, MA, in 1990 and 1995, respectively.

He was a visiting scientist at the Research Laboratory of Electronics, Massachusetts Institute of Technology, Cambridge, MA, between 1992 and 1994, working on femtosecond carrier dynamic studies of semiconductors and metals. He was with the National Science Foundation (NSF) Center of Quantized Electronics Structures (QUEST), University of California, Santa Barbara, CA, from 1995 to 1996 as an assistant research engineer, conducting research on quantum dots, GaN, microcavity, high-speed communication devices and systems. In 1996, he joined the Graduate Institute of Electro-Optical Engineering and Department of Electrical Engineering at National Taiwan University, Taipei, Taiwan, R.O.C., where he is now a full professor. He founded the Ultrafast Optics Group and his research interest is primarily concerned with nano-acoustics, femtosecond laser technology, THz optoelectronics, and biomedical optics.

Dr. Sun is a member of the American Physical Society, a senior member of the IEEE Laser and Electro-Optics Society, a fellow of the Optical Society of America, and a fellow of the Royal Microscopical Society. He was the recipient of year 2000 C.N. Yang Outstanding Young Researcher Award from Association of Asian Pacific Physical Society and the 2001 Academia Sinica Research Award for Junior Researchers from Academia Sinica of Taiwan. He was also the recipient of the 2004 Distinguished Research Achievement Award from National Taiwan University and the 2004 Distinguished Research Award from National Science Council of Taiwan. 